\title{
Bone bruise
}

Trauma Berufskrankh 2006 - 8[Suppl 2]:

S182-S187

DOI 10.1007/s10039-006-1153-8

Online publiziert: 24. Juni 2006

○) Springer Medizin Verlag 2006

H.-J. Böhm · L. W. Poll

BG-Unfallklinik GbR, Duisburg

\section{Bone bruise in der MRT}

\section{Fallberichte}

Traumatische Knochenmarködeme (Bone bruise) sind in der Magnetresonanztomographie (MRT) mit hoher Sensitivität zu erfassen. Durch die inzwischen flächendeckende Verfügbarkeit von MRScannern werden sie in der Diagnostik am Bewegungsapparat bei verschiedenen
Verletzungen und Erkrankungen immer häufiger gefunden $[1,2,4,5,9]$.

Das Knochenmarködem stellt eine unspezifische Reaktion des Knochens
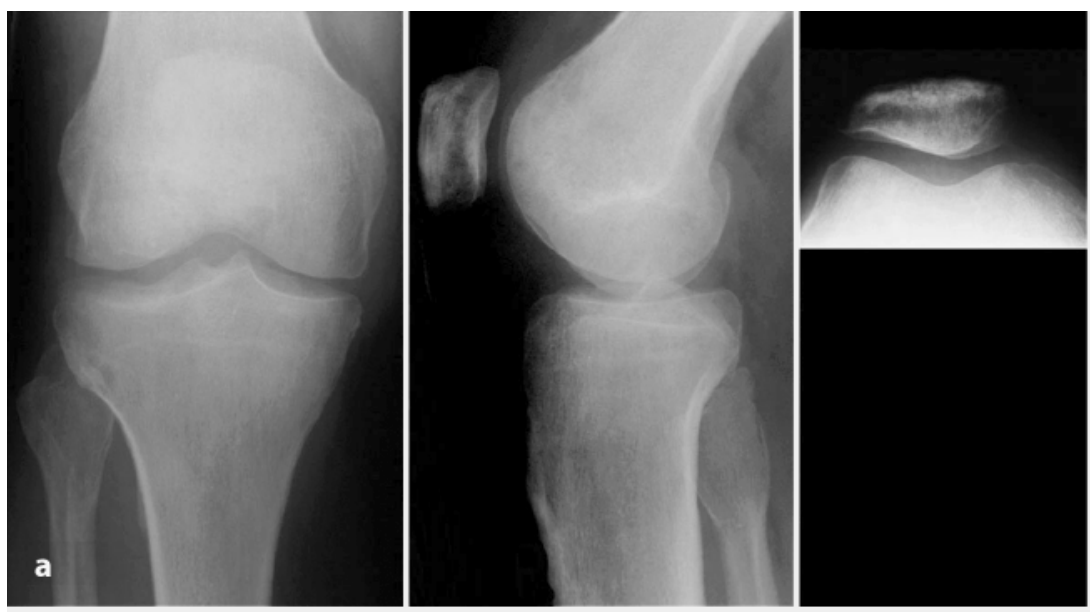

Abb. 14 Fall 1, Beispiel für Bone bruise nach Kniegelenkdistorsion und zweimaliger Arthroskopie

dar. Die Ursachen hierfür können vielfältig sein. Sie reichen von Entzündungen, Tumoren, Arthrosen und Osteonekrosen bis hin zur traumatischen Mikrofrakturierung, die mit konventionell radiologischen Verfahren nicht zu erfassen ist.

Der Begriff Bone bruise wird in der Regel im Zusammenhang mit posttraumatischen Zuständen im Sinne von Spongiosafrakturen verwendet. Eine Übersichtsarbeit hierzu mit Gegenüberstellung von MRT- und histologischen Befunden findet sich bei Rangger et al. [7]. Festzuhalten ist, dass ein Bone bruise nicht nur durch ein singuläres Trauma, sondern vielmehr auch durch chronisch-repetitive Einwirkungen verursacht werden kann [3]. Veränderungen im Sinne eines Bone bruise werden nicht nur an den Extremitäten gefunden, sondern sind auch für die Wirbelkörper beschrieben $[8,10]$.

Die nachfolgenden Kasuistiken von Knochenmarködemen sollen illustrieren, dass ein posttraumatisch entstandenes Bone bruise im Knochen durch chronische, u. U. auch persistierende Schmerzen am Bewegungsapparat einen eigenen Krankheitswert besitzen kann [5]. Dieser Aspekt erscheint uns aus traumatologischer Sicht mitteilenswert, da bei persistierenden Beschwerden und ansonsten negativen Befunden in der Nativradiologie häufig nur der MRT-Befund des Bone bruise den Weg zur entsprechenden Therapie weist.

\section{Kasuistiken}

\section{Fall 1}

Ein 39-jähriger Mann erlitt eine Verstauchung des Kniegelenks im Sinne eines Valgustraumas. Klinisch bestand eine Innenmeniskussymptomatik mit Einklemmungszeichen und Streckdefizit. Bei einer frühzeitig durchgeführten Kniegelenkarthroskopie 2 Tage nach dem Ereignis zeigten sich ein Korbhenkelriss des Innenmeniskus sowie eine Teilruptur des vorderen Kreuzbands bei klinisch suffizienter Stabilität. Nach Refixation des Innenmeniskus persistierten die Beschwerden im Sinne von belastungsabhängigen Schmerzen und Schwellneigung. 3 Monate nach dem Ereignis erfolgte die Rearthroskopie mit Knorpelglättung an der medialen Oberschenkelrolle und Resektion einer Plica mediopatellaris, wonach es jedoch immer noch nicht zur Beschwerdefreiheit kam.

Das Nativröntgenbild zeigt im Wesentlichen einen altersentsprechenden Normalbefund ( $\bullet$ Abb. 1a). In der anschließend durchgeführten Kniegelenk-MRT zeigte sich ein Bone bruise des lateralen Femurkondylus, des lateralen Tibiakopfs sowie der zentralen Anteile des Tibiaplateaus (• Abb. 1b). Das zusätzlich durchgeführte CT der Kniegelenkregion zeigte multiple Osteolysen in den Femurkondylen, im Tibiaplateau keine Auffälligkeiten (- Abb. 1c). Nach vorübergehender Entlastung und anschließendem beschwerdeadaptiertem Belastungsaufbau konnte ein vollständiger Rückgang der Beschwerden erreicht werden.

Resümee. Die Pathogenese des Bone bruise ist im vorliegenden Fall nicht genau zu eruieren. Ursächlich kommen hier sowohl das Trauma selbst als auch die durchgeführten Arthroskopien in Betracht. Die in der CT-Untersuchung festgestellten Osteolysen sind am ehesten als Inaktivitätsosteopenie zu werten.

\section{Fall 2}

Ein 43 Jahre alter Mann zog sich bei einem Motorradsturz eine Schultereckgelenksprengung links sowie eine Prellung der Medialseite des linken Kniegelenks zu. Die Anamneseerhebung ergab keine Verstauchung des Kniegelenks. Die Behandlung beider Verletzungen war konservativ-funktionell.

Bei persistierenden erheblichen Beschwerden an der Innenseite des Kniegelenks erfolgte nach 14 Tagen eine weiterführende Diagnostik. Initial zeigte sich im Nativröntgenbild des Kniegelenks kein pathologischer Befund ( $\bullet$ Abb. 2a). Das MRT ergab ein subchondrales Bone bruise des medialen Femurkondylus sowie im zentralen ventralen Anteil des Tibiaplateaus ( $\bullet$ Abb. 2b). Zudem fanden sich ein mediales periartikuläres Weichteilödem und ein Begleiterguss. Unter temporärer Entlastung über 4 Wochen und sukzessivem Belastungsaufbau kam es zum vollständigen Rückgang der Beschwerden.
Trauma Berufskrankh 2006 - 8[Suppl 2]:

S182-S187

DOI 10.1007/s10039-006-1153-8

(c) Springer Medizin Verlag 2006

H.-J. Böhm · L. W. Poll

\section{Bone bruise in der MRT. Fallberichte}

\section{Zusammenfassung}

Das posttraumatische Knochenmarködem (Bone bruise) findet sich bei ansonsten negativen radiologischen Untersuchungsergebnissen als einziges Symptom, durch das sich chronisch persistierende Extremitätenschmerzen erklären lassen. Für seine Entstehung kommen verschiedenste Ursachen in Frage. Dies wird anhand mehrerer Fallberichte mit jeweils unterschiedlichem Schwerpunkt dargestellt. Sie heben die Wertigkeit der MRT-Untersuchung für die Diagnostik posttraumatischer Zustände hervor.

\section{Schlüsselwörter}

Bone bruise - Knochenmarködem · Trauma . Fallbericht $\cdot$ MRT

\section{Bone bruise as revealed by MRI. Case reports}

\begin{abstract}
When the results of radiological investigations are otherwise negative, posttraumatic bone marrow oedema (bone bruise) becomes apparent as the only explanation for chronic persistent pain in the extremities. A number of different reasons can be considered for it, as illustrated in this presentation of several case reports, each with a different emphasis. They make it obvious how important MRI is in the diagnosis of posttraumatic conditions.
\end{abstract}

\section{Keywords}

Bone bruise - Bone marrow oedema · Trau$\mathrm{ma} \cdot$ Case report $\cdot$ MRI 


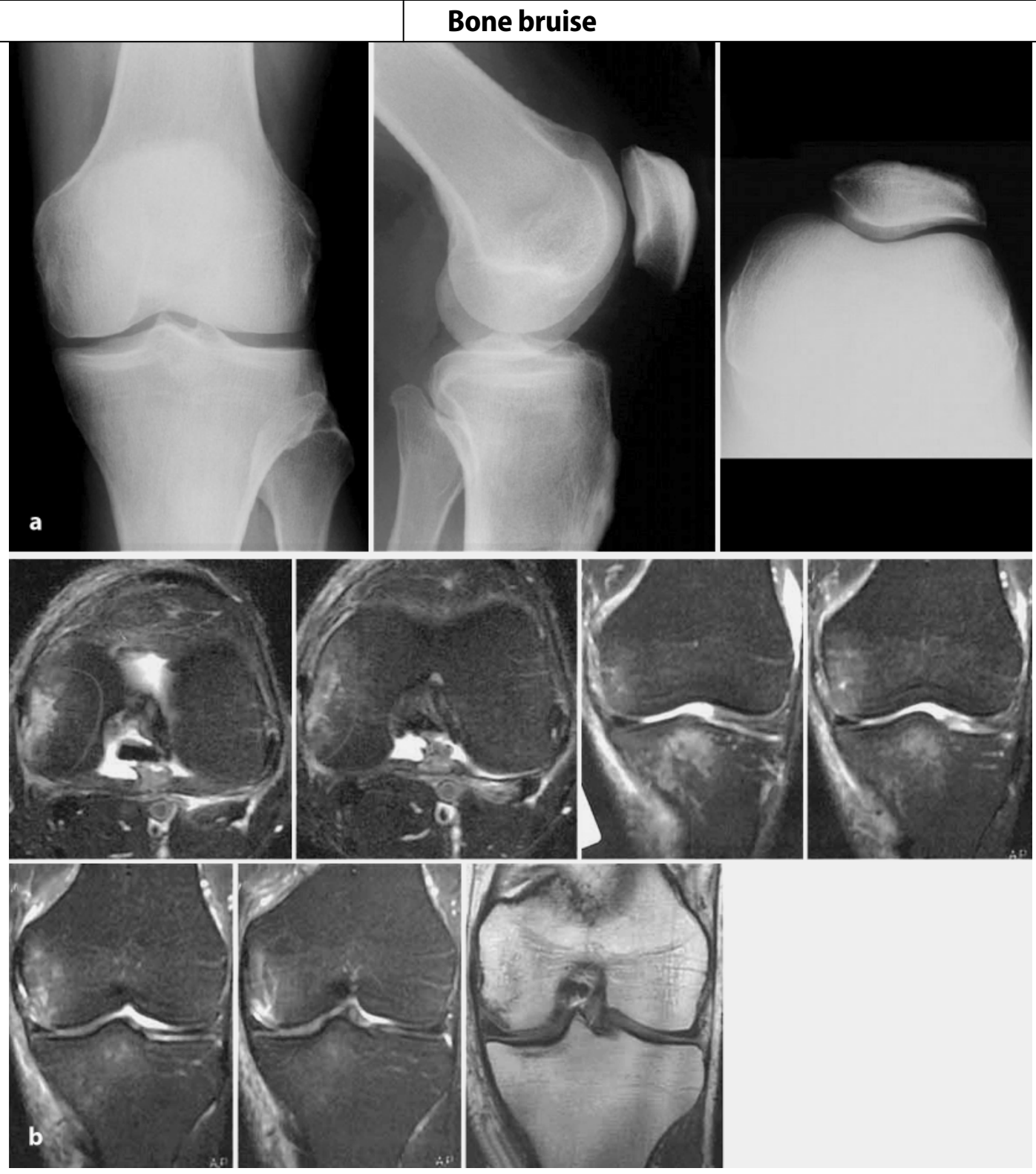

Abb. 24 Fall 2, direkte Anprallverletzung der Medialseite des Kniegelenks mit Bone bruise im medialen Femurkondylus und im Tibiakopf

Resümee. Durch das direkte Anpralltrauma hatte sich dieser Patient eine Mikrofrakturierung an der medialen Oberschenkelrolle mit Begleitödem der Weichteile zugezogen. Diese Veränderung war im konventionellen Röntgenbild nicht zu erfassen.

\section{Fall 3}

Eine 35-jährige Frau zog sich eine Quetschung der Fußwurzel zu. Im Nativröntgenbild ergab sich kein pathologischer Befund (- Abb. 3a).3 Wochen später wurde wegen Beschwerdepersistenz eine MRTUntersuchung vorgenommen. Hierbei fanden sich ein ausgeprägtes Bone bruise im vorderen Abschnitt des Kalkaneus sowie begleitend eine plantare Weichteil- schwellung. Da in der MRT (• Abb. 3b, c) der Verdacht auf das Vorliegen von Frakturlinien bestand (in T1-gewichteter Sequenz), erfolgte zudem eine CT-Untersuchung des Kalkaneus, bei der sich keine Frakturlinien zeigten (• Abb. 3d).

Resümee. Auch das CT ist in Fällen von Spongiosafrakturen nicht sensitiv genug, um eine Störung der Knochentextur durch Mikrofrakturierung zu detektieren.

\section{Fall 4}

Eine 22 Jahre alte Frau mit einem vorausgegangenen und operierten Sprunggelenkverrenkungsbruch links zog sich ein Supinationstrauma des rechten Sprunggelenks zu. In der Folgezeit kam es unter konservativer Therapie zu massiven, persistierenden Beschwerden. Im Nativröntgenbild zeigte sich kein pathologischer Befund am rechten oberen Sprunggelenk (• Abb. 4a). 5 Wochen nach dem Ereignis erfolgte eine MRT-Untersuchung, bei der sich neben einem Erguss im oberen Sprunggelenk ein massives Bone bruise des Talus zeigte ( $\bullet$ Abb. 4b, c). Entscheidend war jedoch hier der klinische Aspekt. Es bestand eine ausgeprägte Symptomatik einer Algodystrophie (ロ Abb. 4d), nach entsprechender Behandlung waren auch hier die Beschwerden rückläufig.

Resümee. Die Ergebnisse der bildgebenden Verfahren müssen immer im Kontext des klinischen Befunds interpretiert werden. Führend war in diesem 



Abb. 3 ム Fall 3, ausgeprägtes Bone bruise im ventralen Anteil des Kalkaneus nach Quetschverletzung, Röntgen und CT unauffällig

Fall die Symptomatik der Algodystrophie, die auch für den therapeutischen Ansatz maßgebend war.

\section{Fall 5}

Ein 71 Jahre alter Mann klagte zum Zeitpunkt der Erstvorstellung über seit $14 \mathrm{Ta}$ gen bestehende Knieschmerzen lateral, wobei in der Anamnese kein Trauma zu erheben war. Die Beschwerden waren unter Beugung stärker als in Streckstellung. Im Nativröntgenbild zeigte sich ein altersentsprechender Normalbefund (- Abb. 5a). In der MRT-Untersuchung bot sich das Bild eines ausgeprägten Knochenmarködems des lateralen Femurkondylus mit begleitendem Weichteilödem (- Abb. 5b, c), somit wurde eine primäre Osteonekrose diagnostiziert.
Resümee. Neben traumatisch verursachtem Bone bruise kommen für ein Knochenmarködem eine Reihe anderer Ursachen in Frage. Hier sind Anamneseerhebung und klinischer Befund zielführend.

\section{Fall 6}

Bei dieser 54-jährigen Frau bestanden in der Anamnese keine starken körperlichen Belastungen im Sport, normalerweise wurden keine langen Gehstrecken zurückgelegt. 3 Wochen vor der Konsultation hatte die Patientin eine Bergwanderung unternommen, wenige Tage später traten belastungsabhängige Schmerzen in der Ferse und eine Schwellung an der Knöchelgabel und am Rückfuß auf.

Im Nativröntgenbild und im CT des Fersenbeins ergab sich kein pathologischer Befund. In der daraufhin durch- geführten MRT zeigte sich ein diffuses, netzförmiges Bone bruise im gesamten Kalkaneus (- Abb. 6a, b). Es wurde konservativ mit 6-wöchiger, kompletter Entlastung behandelt. Danach bildeten sich die Beschwerden vollständig zurück.

\section{Fazit}

Die Falldarstellungen demonstrieren, dass die Diagnose eines Bone bruise durchaus als relevant zu werten ist. Häufig stellt dieser Befund die einzig fassbare pathologische Veränderung dar. Wenn dies mit den Beschwerden des $\mathrm{Pa}$ tienten korreliert, ist eine weiterführende Diagnostik nicht mehr notwendig. Da bei diesen posttraumatischen Zuständen mit Einblutungen und Mikrofrakturen im spongiösen Bereich keine Kortikalisunterbrechung oder gar Dislokati- 


\section{Bone bruise}


Abb. 4 ム Fall 4, Bone bruise des Talus nach Supinationstrauma, klinisches Bild einer Algodystrophie im Vordergrund stehend


Abb. 5 \ Fall 5, Osteonekrose der lateralen Femurrolle 



Abb. $6 \Delta$ Fall 6, ausgedehntes Bone bruise im gesamten Kalkaneus, bei ansonsten leerer Anamnese als durch Überlastung bedingt interpretiert

on vorliegt, wird in der Regel konservativ behandelt, was mit mehrwöchiger Entlastung verbunden ist. Zur Dokumentation des Therapieerfolgs und zum Ausschluss möglicher Komplikationen $[2,4]$ halten wir eine Kontroll-MRT im Verlauf für sinnvoll.

\section{Korrespondierender Autor}

Dr. H.-J. Böhm

BG-Unfallklinik GbR

Großenbaumer Allee 250, 47249 Duisburg

boehm@mac.com

Interessenkonflikt. Es besteht kein Interessenkonflikt. Der korrespondierende Autor versichert, dass keine Verbindungen mit einer Firma, deren Produkt in dem Artikel genannt ist, oder einer Firma, die ein Konkurrenzprodukt vertreibt, bestehen. Die Präsentation des Themas ist unabhängig und die Darstellung der Inhalte produktneutral.

\section{Literatur}

1. Alanen V, Taimela S, Kinnunen J et al. (1998) Incidence and clinical significance of bone bruises after supination injury of the ankle. J Bone Joint Surg Br 80-B: 513-515

2. Bohndorf K, Imhof H, Schibany N (2001) Bildgebende Diagnostik akuter und chronischer osteochondraler Läsionen am Talus. Orthopäde 30: 1219

3. Dienst M, Blauth M (2000) Bone bruise of the calcaneus. Clin Orthop 378: 202-205

4. Krappel FA, Bauer E, Harland U (2005) Are bone bruise a possible cause of osteochondritis dissecans of the capitellum? A case report and review of the literature. Arch Orthop Trauma Surg 125: 545-549

5. Krause R, Mayerhofer K, Glas K (1998) Verlauf und Therapie eines posttraumatischen Marködems in der proximalen Tibia. Sportorthop Sporttraumatol 14: 122-125

6. Radke S, Vispo-Seara J, Walther M et al. (2001)

Transient bone marrow oedema of the foot. Int Orthop 25: 263-267
7. Rangger C, Kathrein A, Freund MC et al. (1998) Bone bruise of the knee. Acta Orthop Scand 69: 291 294

8. Scheunemann D, Lehmann W, Briem D et al. (2005) Zur Bedeutung der MRT-Diagnose „bone bruise" bei kindlichen Wirbelsäulenverletzungen. Unfallchirurg 108: 638-644

9. Stein LN, Fischer DA, Fritts HM et al. (1995) Occult osseous lesions associated with anterior cruciate ligament tears. Clin Orthop 313: 187-193

10. Teli M, Roeck N de, Horowitz MD et al. (2005) Radiographic outcome of vertebral bone bruise associated with fracture of the thoracic and lumbar spine in adults. Eur Spine J 14: 541-545 